\title{
FREE-SURFACE FLOW DUE TO A SOURCE SUBMERGED IN A FLUID OF INFINITE DEPTH WITH TWO STAGNANT REGIONS
}

\author{
HOCINE MEKIAS ${ }^{1}$ and JEAN-MARC VANDEN-BROECK ${ }^{1}$
}

(Received 9 July 1991; revised 2 January 1992)

\begin{abstract}
Two-dimensional free-surface flows produced by a submerged source in a fluid of infinite depth are considered. It is assumed that the point on the free surface just above the source is a stagnation point and that the fluid outside two shear layers is at rest. The free-surface profile and the shape of the shear layers are determined numerically by using a series-truncation method. It is shown that there is a solution for each value of the Froude number $F>0$. When $F$ tends to infinity, the flow also describes a thin jet impinging in a fluid at rest.
\end{abstract}

\section{Introduction}

Free-surface flows due to a submerged source have been the subject of many investigations. Some authors ([1], [2], [7], [8]) assumed that the free surface just above the source is cusplike, whereas others ([4], [5], [6]) assumed that the point just above the source on the free surface is a stagnation point. Various geometric configurations (finite depth, sloping bottom, and infinite depth) have been considered.

In this paper, we consider the steady two-dimensional potential flow due to a source beneath a free surface in water of infinite depth. We assume that the point just above the source is a stagnation point and the fluid outside two shear layers $B I$ and $B^{\prime} I^{\prime}$ is at rest (see Figure 1). Far below the source the velocity is uniform.

\footnotetext{
${ }^{1}$ Dept. of Math. and Center for Math. Sciences, University of Wisconsin-Madison, WI 53705.

(C) Copyright Australian Mathematical Society 1992, Serial-fee code 0334-2700/92
} 
This model of flow was proposed by Peregine [6] when he studied a line source beneath a free surface; however he did not compute it. To calculate this flow, we adopt a series-truncation technique similar to the one described by Vanden-Broeck and Keller [8], and Mekias and Vanden-Broeck [4].

As we shall see, the problem is characterised by the Froude number

$$
F=Q / g^{1 / 2} H^{3 / 2} \text {. }
$$

Here, $2 Q$ is the strength of the source, $g$ is the acceleration of gravity, and $2 H$ is the total width of the fluid in motion far below the source. A solution exists for each value of $F>0$. For small $F$, the source is below the freesurface level of the static fluid. As $F$ tends to infinity, the source moves upwards and eventually rises above the free-surface level of the static fluid. In the latter case, the lower part of the flow describes a thin jet impinging in a fluid at rest.

The problem is formulated in Section 2, the numerical scheme is described in Section 3, and the results are discussed in Section 4.

\section{Formulation}

We consider the fluid flow due to a source $S$ of strength $2 Q$ and located at the origin of the coordinates (see Figure 1). We assume that the fluid is inviscid and incompressible, and the flow is irrotational. The point $\mathrm{C}$, on the free surface $B^{\prime} C B$ and just above the source, is a stagnation point. The surfaces $B I$ and $B^{\prime} I^{\prime}$ separate the dynamic fluid from the static one. The $x$ axis is parallel to the level of the free surface of the regions at rest, and the $y$-axis is through the source. Far below the source, the flow is uniform with velocity $U_{0}$, and the total width of the flow is $2 H$. Because of the symmetry with respect to the $y$-axis, we shall only consider the right half of the flow. We define dimensionless variables by choosing $\left(Q^{2} / g\right)^{1 / 3}$ as the unit length and $(Q g)^{1 / 3}$ as the unit velocity.

Let $\phi$ and $\psi$ denote the velocity potential and the stream function. The components of the velocity in the $x$ and $y$ directions are denoted by $u$ and $v$, respectively. Since the flow is potential, the complex potential function

$$
f=\phi+i \psi \text {, }
$$

and the complex velocity

$$
\zeta=u-i v,
$$

are analytic functions of the complex variable $z=x+i y$. The kinematic conditions on the streamlines SI and SC are

$$
u=0 \text { and } v<0 \text { on SI, }
$$




$$
u=0 \text { and } v>0 \text { on SC. }
$$

The condition of no pressure jump across the streamline BI yields

$$
g y+\frac{1}{2}\left(u^{2}+v^{2}\right)=g y+\text { constant on } \mathrm{BI} \text {. }
$$

Hence

$$
u^{2}+v^{2}=\text { constant on } \mathrm{BI} \text {. }
$$

The stream function is chosen so that $\psi=0$ on SCBI and $\psi=-1$ on SI. Without loss of generality, we choose $\phi=0$ at $\mathrm{C}$. In the $f$-plane, the flow domain is the strip $-1 \leq \psi \leq 0$.

The transformation

$$
f=\frac{1}{\pi} \log \left\{\frac{B\left(1+t^{2}\right)^{2}-\left(1-t^{2}\right)^{2}}{4 t^{2}}\right\}, \quad B>1,
$$

maps the flow domain onto the quarter of the unit circle in the first quadrant

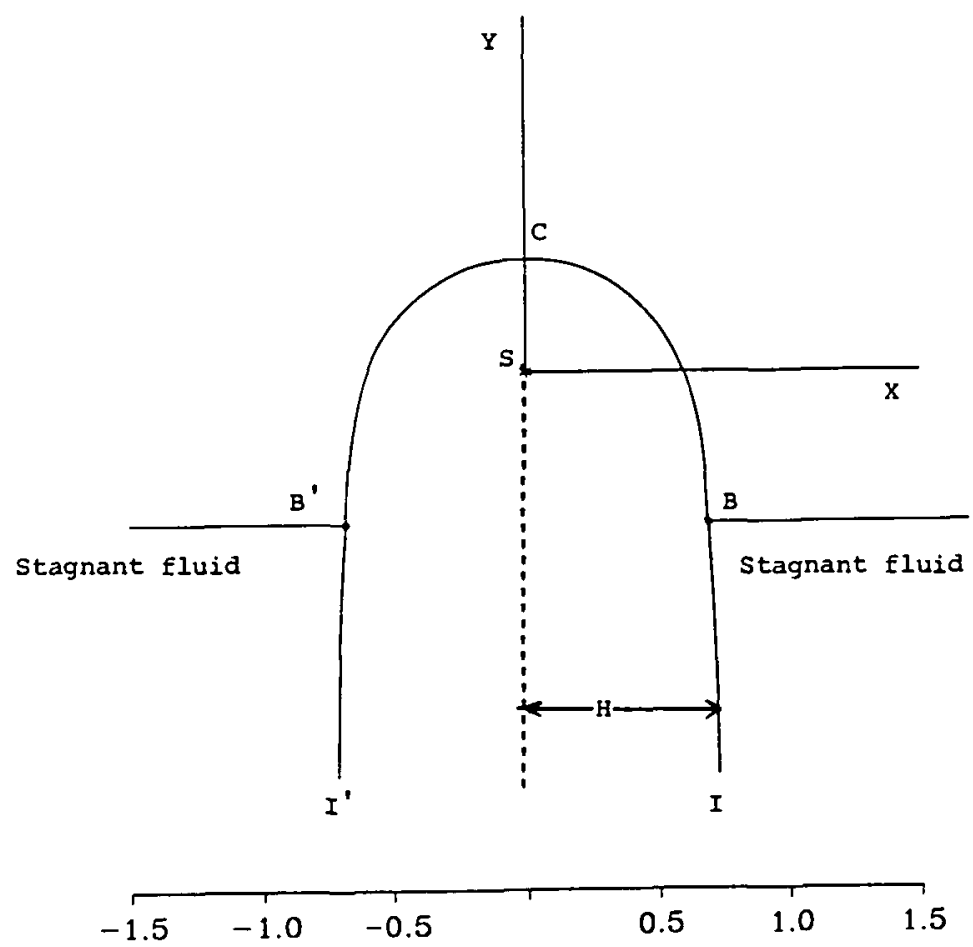

FIgure 1. Typical flow with infinite depth and two regions at rest in the far field. The source is at the origin, the $x$-axis is parallel to the level of the free surface of the regions at rest, and the $y$-axis is through the source. The figure is an actual computed profile for $B=30$ and $F=1.7$. 
of the $t$-plane. The point I far below the source, the source $\mathrm{S}$, the stagnation point $\mathrm{C}$, and the point $\mathrm{B}$ are mapped onto the origin, the point $t=i b$, $(0<b<1)$, the point $t=i$, and the point $t=1$, respectively. Thus, the portion $\mathrm{CB}$ of the free surface is mapped onto the circular arc (see Figure 2). Here $b=(\sqrt{B}-1) /(\sqrt{B}+1)^{1 / 2}$.

We seek the complex velocity $\zeta(t)=u-i v$ as a series in powers of the variable $t$. But first, we should determine the local behaviours of $\zeta(t)$ at the singularities, $t=i b$ and $t=i$, which correspond to the source at $S$ and to the stagnation point at $C$, respectively.

Local analysis shows that the behaviour near the source is

$$
\zeta=O\left(\frac{1}{t-i b}\right) \text { as } t \rightarrow i b,
$$

and that the behaviour inside the right angle at $C$ is

$$
\zeta=O(t-i) \text { as } t \rightarrow i .
$$

From the choice of the dimensionless variables, it follows that the Froude number $F$ defined by (1) is given by

$$
F=[\zeta(0)]^{3 / 2} \text {. }
$$

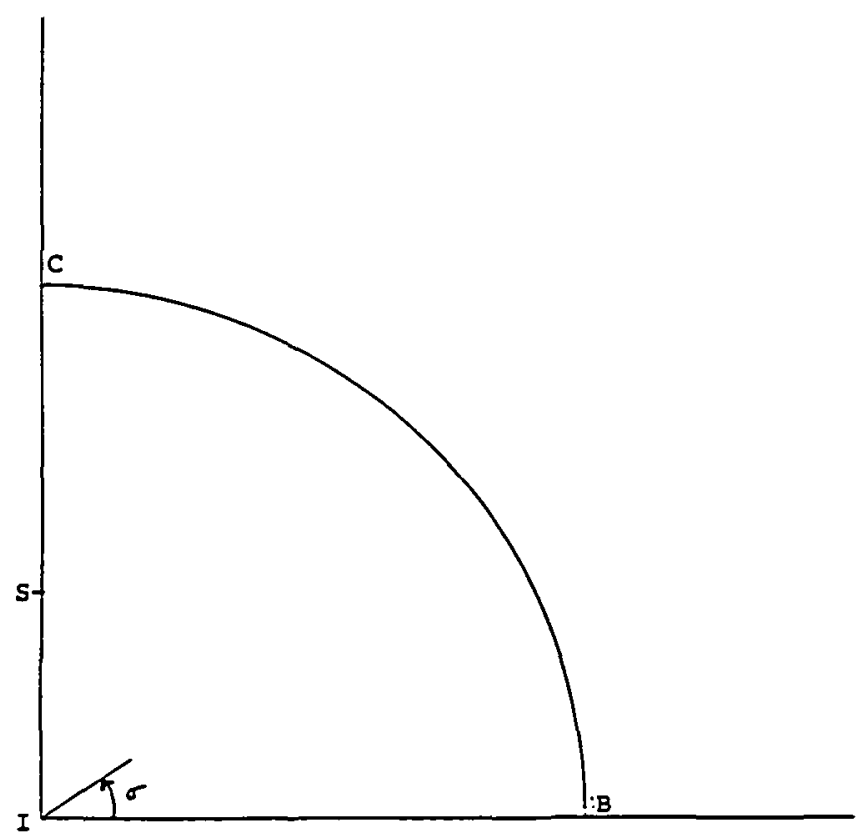

Figure 2. The complex $t$-plane. 
Now that we know the local behavior of $\zeta(t)$ near the singularities, we write

$$
\zeta(t)=i A \frac{(t-i)(t+i b)}{(t+i)(t-i b)} \exp \left(\sum_{n=1}^{\infty} a_{n}(i t)^{2 n-1}\right) .
$$

The coefficients $a_{n}$ and $A$ are to be found. Since (8) satisfies (5) and (6), we expect the series to converge in the quarter disc in the $t$-plane. The coefficients $a_{n}$ and $A$ are chosen to be real so that the boundary conditions $(2 a),(2 b)$, and (3) are satisfied.

In terms of the dimensionless variables, the Bernoulli equation on the free surface $\mathrm{CB}$ is

$$
|\zeta|^{2}+2 y=\text { const }
$$

In the $t$-plane, it must hold on the circular arc $t=e^{i \sigma}, 0<\sigma<\pi / 2$. Differentiating (9) with respect to $\sigma$ and using the identity

$$
\frac{\partial x}{\partial \phi}+i \frac{\partial y}{\partial \phi}=\frac{1}{\zeta}
$$

we rewrite the Bernoulli equation (9) as

$$
\frac{\partial|\zeta|^{2}}{\partial \sigma}-\frac{4}{\pi}\left[\frac{\sin (2 \sigma)}{\cos (2 \sigma)+(B+1) /(B-1)}\right] \frac{v}{u^{2}+v^{2}}=0
$$

Equation (11) must hold on the circular arc CB. By setting $t=e^{i \sigma}(0<\sigma<$ $\pi / 2)$ in (8), we get $\zeta\left(e^{i \sigma}\right)$. Substituting that expression into (11), we obtain an equation to determine the coefficients $a_{n}$ and $A$.

\section{Numerical solution}

We truncate the series (8) after $N$ terms. The $N$ coefficients $a_{n}$ and the constant $A$ are found by collocation. Thus, we introduce $N+1$ mesh points:

$$
\sigma_{I}=\frac{\pi}{2(N+1)}\left(I-\frac{1}{2}\right), \quad I=1, \ldots, N+1 .
$$

By using (8), we obtain $\zeta$ and $\partial|\zeta|^{2} / \partial \sigma$ at the mesh points $\sigma_{I}$ in terms of the coefficients $a_{n}, B$ and $A$. Upon substituting these expressions into 
(11), we obtain $N+1$ nonlinear algebraic equations for the $N+2$ unknowns $\left\{a_{n}\right\}_{n=1}^{N}, A$ and $B$. The $(N+2)^{t h}$ equation is obtained by fixing the value of the potential function $\phi$ at the point $B$ (i.e., we specify a value of $B$ ). The resulting $N+1$ equations are solved by using Newton's method. Once the coefficients $\left\{a_{n}\right\}_{n=1}^{N}$ and $A$ are known, the position of the stagnation point $\mathrm{C}$, the shape of the free surface, and the shape of the separating streamline BI are obtained by integrating numerically (10) along the imaginary radius from $t=i b$ to $t=i$, along the circular arc, $0 \leq \sigma \leq \pi / 2$, and along the real radius from $t=1$ to $t=0$.

We use the numerical procedure described above to compute solutions for various values of $B>1$. The coefficients decrease very rapidly as $N$ increases. Table 1 shows some of the coefficients of the series (8) and the corresponding Froude number $F$ (calculated from (7)) for different values of $B$. Most of the results presented here are obtained with $N=40$.

Typical profiles for $B=30, B=1.3, B=5, B=10$ and $B=50$ are shown in Figure 1, Figure 3a, Figure 3b, Figure 3c and Figure 3d, respectively. For $B \sim 1$, but $B>1$, the source lies far below the level of the free surface of the fluid at rest and the free surface is very close to the horizontal level, as in Figure 3a. As $B$ increases, the source moves upwards, and eventually, is above the level of the free surface of the fluid at rest. In Figure 4, we present values of the Froude number $F$ versus $B$. As $B \rightarrow \infty, F \rightarrow \infty$ and the flow near the stagnation point approaches a limiting configuration with a falling jet. This limiting flow configuration was calculated by Mekias and Vanden-Broeck [4] (see Figure 5). For $B=1, F=0$ and the level of the free surface is $y=\infty$. The corresponding solution is simply the flow due to a submerged source in an unbounded fluid, i.e. $f=(1 / \pi) \log z$.

Finally, let us mention that the family of solutions described in this paper does not include as particular cases the solution with cusp of Tuck and Vanden-Broeck [7] or the solutions with stagnation points of Hocking and Forbes [3] and of Mekias and Vanden-Broeck [5]. Therefore it would appear that different solutions are possible for the same value of the Froude number.

TABLE 1. Some values of the coefficients of the series (6) and the Froude number $F$ for some values of $B$.

$\begin{array}{cccccc}B & F & a_{1} & a_{10} & a_{30} & a_{40} \\ 1.3 & .165 & -0.369 & 9.0410^{-5} & 2.0410^{-6} & -2.4010^{-7} \\ 10 . & 1.35 & 0.173 & 1.4510^{-4} & 1.2110^{-6} & -1.5010^{-7} \\ 20 . & 1.58 & 0.203 & 6.1910^{-4} & 9.6710^{-7} & -1.1810^{-7} \\ 30 . & 1.70 & 0.210 & 1.3010^{-3} & 1.1110^{-6} & -1.0310^{-7} \\ 45 . & 1.82 & 0.213 & 2.3910^{-3} & 2.8110^{-6} & -7.7810^{-8}\end{array}$


(a)

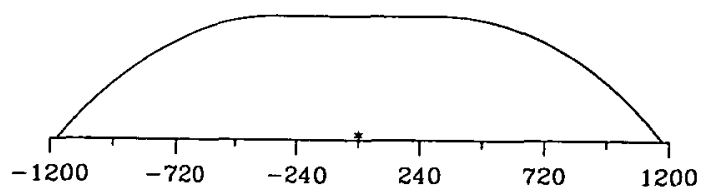

(b)
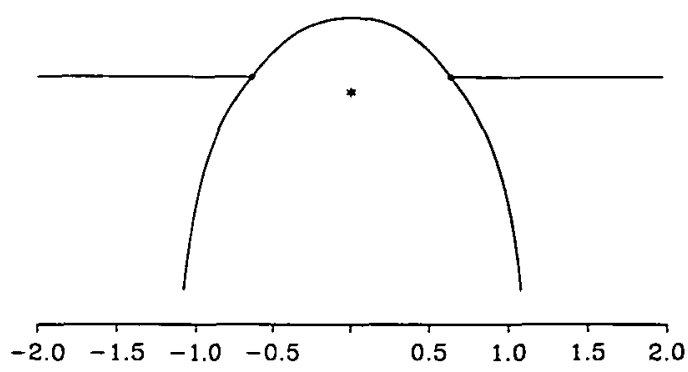

(c)

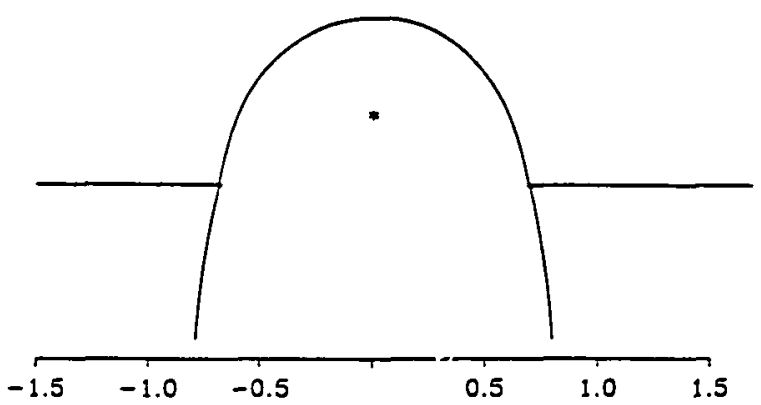

(d)

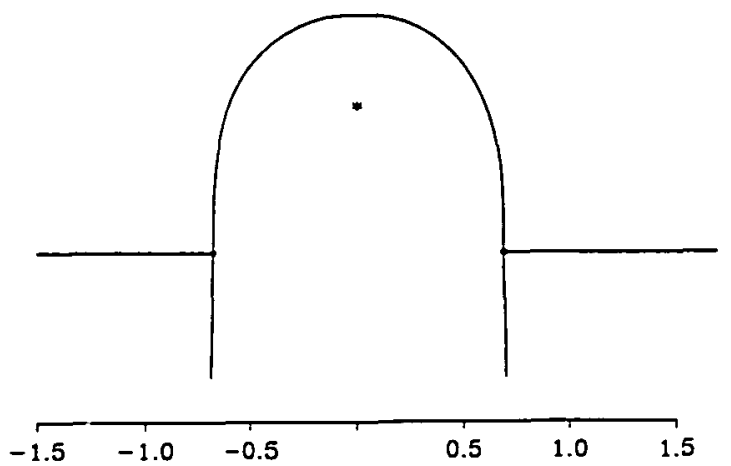

Figure 3. Typical free surface profiles. The star indicates the position of the source and the horizontal lines are the level of the free surface of the fluid at rest. The vertical scale is the same as the horizontal scale. (a) $B=1.3, F=.165$. (b) $B=5, F=.7$. (c) $B=10, F=1.35$. (d) $B=50, F=1.87$. 


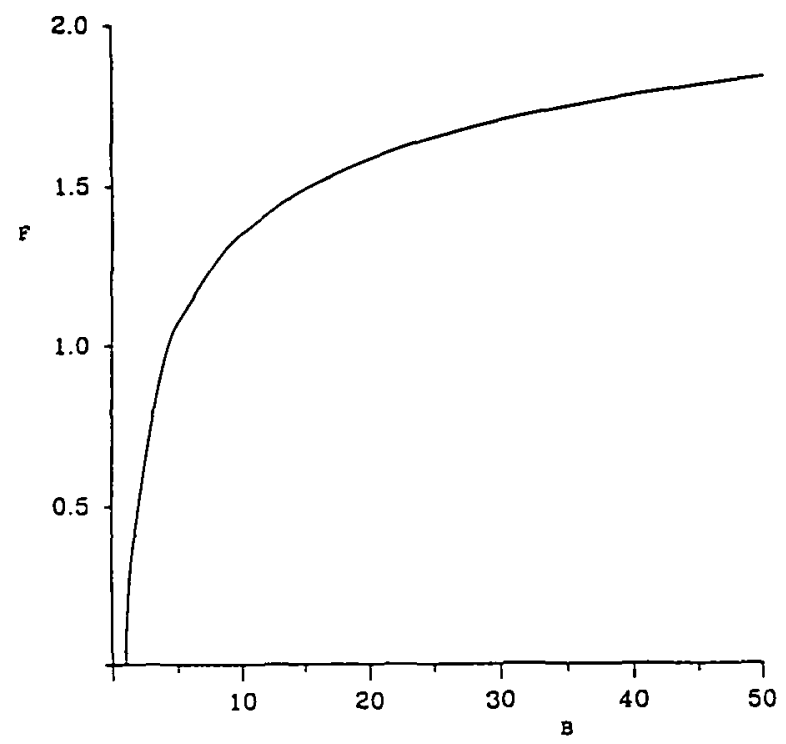

Figure 4. The Froude number $F$ vs. $B$.

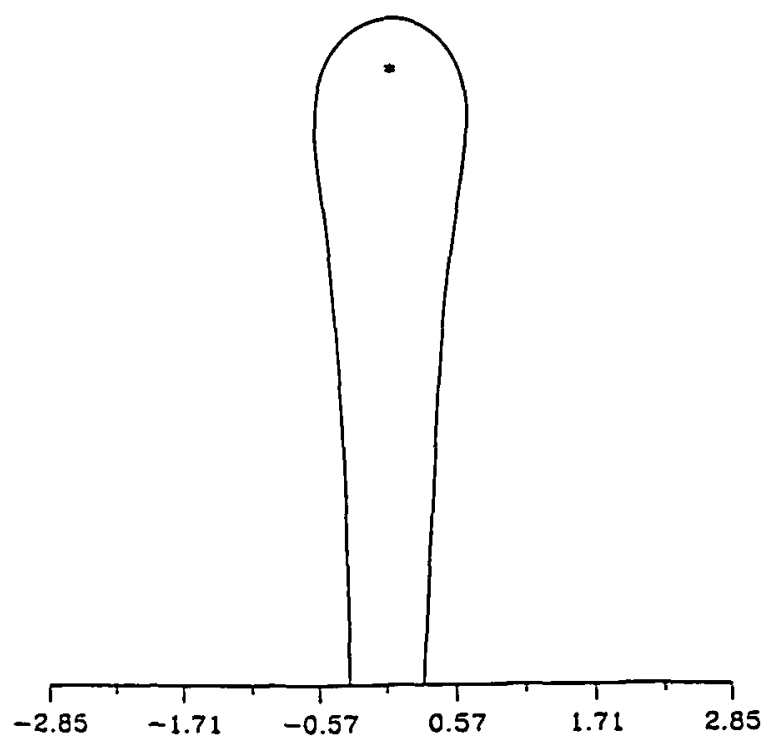

Figure 5. The limiting configuration as $B \rightarrow \infty$. The star indicates the position of the source. As $y \rightarrow-\infty$, the flow approaches a thin vertical jet. The vertical scale is the same as the horizontal scale. (Figure from Mekias and Vanden-Broeck [5].) 


\section{References}

[1] A. Craya, "Theoretical research on the flow of non-homogeneous fluids", La Houille Blanche 4 (1949) 44-55.

[2] G. C. Hocking, "Cusp-like free-surface flows due to a submerged source or sink in the presence of a flat or sloping bottom", J. Austral. Math. Soc. B 26 (1985) 470-486.

[3] G. C. Hocking and L. K. Forbes, "A note on the flow induced by a line sink beneath a free surface", J. Austral. Math. Soc. B 32 (1991) 251-260.

[4] H. Mekias and J.-M. Vanden-Broeck, "Supercritical free-surface flow with a stagnation point due to a submerged source", Phys. Fluids A 1 (1989) 1694-1697.

[5] H. Mekias and J.-M. Vanden-Broeck, "Subcritical flow with a stagnation point due to a source beneath a free surface", Phys. Fluids A 3 (1991) 2652.

[6] D. H. Peregrine, "A line source beneath a free surface", Univ. Wisconsin Math. Res. Center Tech. Summ. Report 1248 (1972).

[7] E. O. Tuck and J.-M. Vanden-Broeck, "A cusp-like free-surface flow due to a submerged source or sink", J. Austral. Math. Soc. B 25 (1984) 443-450.

[8] J.-M. Vanden-Broeck and J. B. Keller, “Free surface flow due to a sink", J. Fluid Mech. 175 (1987) 109-117. 